\title{
Aisthesis
}

Firenze University Press

www.fupress.com/aisthesis

OPEN ACCESS

Citation: F. Valagussa (2021) Lo spirito sfiduciato. Stupidità soggettiva e intelligenza oggettiva. Aisthesis 14(1): 109-115. doi: 10.36253/Aisthesis-12003

Copyright: ( $2021 \mathrm{~F}$. Valagussa. This is an open access, peer-reviewed article published by Firenze University Press (http://www.fupress.com/aisthesis) and distributed under the terms of the Creative Commons Attribution License, which permits unrestricted use, distribution, and reproduction in any medium, provided the original author and source are credited.

Data Availability Statement: All relevant data are within the paper and its Supporting Information files.

Competing Interests: The authors have declared that no competing interests exist.

\section{The defied spirit. Subjective stupidity and objective intelligence}

\author{
Lo spirito sfiduciato. Stupidità soggettiva e intelligenza \\ oggettiva
}

\author{
Francesco VAlagussa \\ Università Vita-Salute San Raffaele \\ valagussa.francesco@hsr.it
}

\begin{abstract}
The present article analyses the different forms of stupidity as they are presented in the two renowned lectures held by Musil in 1937, in particular by trying to connect them to some passages from The Man Without Qualities. Stupidity cannot be reduced to a simple "inability to understand". It becomes the symptom of a wider crisis involving the whole European tradition: a crisis of trust in human nature that ends up compromising every possible overall vision about culture and life. Valéry arrived to a similar outcome in his The Crisis of The Mind. Significantly, both Musil and Valéry attempted to explain this kind of crisis through the analogy with economic processes: by combining their theses, and taking into account some passages of Simmel's Philosophy of Money, we could say that money has concentrated intelligence and trust within itself, reducing individuals to a condition of stupidity and mutual distrust.
\end{abstract}

Keywords: Musil, Valéry, Simmel, Stupidity, Philosophy of Money.
«Lo sfogo viene introdotto da queste parole: "Insomma, la cosa è troppo stupida". Ma la "cosa" troppo stupida è l'uomo che sta parlando»

(Musil [1986a]: 252).

Gli "esemplari della specie "stupidità" ${ }^{1}$ passati in rassegna da Musil nel corso delle due conferenze tenute a Vienna nel marzo del 1937 si potrebbero ritrovare, più o meno, quasi tutti, ne Gli ultimi giorni dell'umanità: c'è la stupidità che sopisce la diffidenza, disarmandola ${ }^{2}$, quella che lo speculatore di Berlino rimprovera ai vienne-

${ }^{1}$ Cfr. Musil ([1986a]: 235).

${ }^{2}$ Cfr. Musil ([1986a]: 238). 
$\mathrm{si}^{3}$. È una "stupidità astuta", una furberia: quando i rapporti di forza sono sbilanciati, il più debole si finge più stupido di quello che $\mathrm{è}^{4}-$ proprio come il generale austriaco sbigottito di fronte al sottotenente prussiano, pieno di grinta e di organizzazione 5 . Poi troviamo le "stupide vittime" che suscitano impazienza e non di rado eccitano selvaggiamente l'immaginazione di uomini crudeli ${ }^{6}$ : viene in mente il Kaiser Guglielmo II che si diverte a umiliare i propri generali ${ }^{7}$. Anche le perifrasi di coloro che si considerano intelligenti ma non lo dicono ${ }^{8}$ - "Non sono più stupido di un altro", «Non mi faranno passare per stupido» - sono ampiamente rappresentate nel romanzo di Kraus?. Da ultimo ci sarebbe la rozzezza come "prassi della stupidità": "non soltanto essere stupidi, ma agire da stupidi, commettere delle stupidaggini» (Musil [1986a]: 245), come Auffenberg che deve scrivere una cartolina, si dimentica e si dà dello stupido di fronte a Brudermann ${ }^{10}$.

1. I casi citati impediscono di ricondurre le manifestazioni di stupidità a una sorta di generica insufficienza sul piano intellettuale. Una certa limitatezza nel livello di comprensione degli eventi era stata "censita" già nel primo Dopoguerra:

${ }^{3}$ Cfr. Kraus ([2007]: 215): «può succedere soltanto a Vienna, ma che un popolo col quale combattiamo fianco a fianco sopporti una simile stupidaggine, è roba da pazzi, voi viennesi non vi rendete proprio conto che siamo in guerra».

${ }^{4}$ Cfr. Musil ([1986a]: 238).

${ }^{5}$ Cfr. Kraus ([2007]: 270-271): «Vedete signori, potete dir quel che volete contro i tedeschi, ma una cosa bisogna riconoscergli, diciamo la verità, hanno l'organizzazione. [...] Così, invece, quel che ci manca è proprio l'organizzazione. In questo i tedeschi ci superano, diciamo la verità». ${ }^{6}$ Cfr. Musil ([1986a]: 239).

${ }^{7}$ Cfr. Kraus ([2007]: 486): «Guglielmo II: Troppo stupidi ... ah ... questa gente non ha senso dell'umorismo!».

${ }^{8}$ Cfr. Musil ([1986a]: 240).

${ }^{9}$ Cfr. Kraus ([2007]: 375): «Per quanto riguarda gli statisti nemici, non possono essere più stupidi dei nostri, perché una cosa simile non esiste in natura».

${ }^{10}$ Cfr. Kraus ([2007]: 131): «Niente ... che stupido... insomma, debbo scrivergli una cartolina. È da Lublino che lo voglio fare, ma nella confusione della ritirata me ne sono completamente dimenticato». "ci sono mancati i concetti per interiorizzare i vissuti» (Musil [1986b]: 105), nel senso che abbiamo visto di tutto e non ci siamo accorti di nulla. Ci siamo scoperti "ciechi, impotenti, anche se armati di conoscenze e carichi di potere» (Valéry [1994a]: 59), come se le determinazioni di una cosa cominciassero a presentare una gradualità nel trapasso dall'una all'altra che "le rende assolutamente inafferrabili ai nostri concetti, saldamente circoscritti» (Simmel [1997]: 188).

Ad essere travolta è la forma-coscienza e la possibilità di costituire "rappresentazioni" in senso classico di ciò che accade: «non si sa più come raccogliere tutto ciò che si vince alla lotteria dell'esperienza. I risultati parlano tutti insieme» (Valéry [2008]: 94). Alcuni termometri piazzati da Simmel "nei punti giusti" avevano già segnalato febbre alta: l'accumulo veloce di immagini, il brusco contrasto tra impressioni, la logica autonoma e indecifrabile del denaro, così come la crisi di fondazione delle scienze prospettavano un quadro clinico già fortemente compromesso. L'effetto di logoramento prodotto dall'intensificazione della vita nervosa non restituisce il tipo metropolitano sul piano meramente psicologico: accanto alla separazione tra intelletto e strati profondi della personalità ${ }^{11}$, corre in parallelo lo sradicamento delle abitudini - queste armature dell'esperienze vengono aggredite direttamente dai vissuti ${ }^{12}$. L'informazione rade al suolo la saggezza ${ }^{13}$ : la lettura di un quotidiano tira in ballo una quantità di cose che «non basterebbe il cervello di un Leibniz per capirle» (Musil [1997]: vol. I, 244). Imbrogliatissima, piena si sottigliezza metafisica ${ }^{14}$, la merce

\footnotetext{
${ }^{11}$ Cfr. Simmel ([1995]: 36-37): «l'intelletto è la più adattabile delle nostre forze interiori: per venire a patti con $\mathrm{i}$ cambiamenti e i contrasti dei fenomeni non richiede quegli sconvolgimenti e quei drammi interiori che la sentimentalità a causa della sua natura conservatrice, richiederebbe necessariamente per adattarsi a un ritmo analogo di esperienze».

${ }^{12}$ Cfr. Simmel ([1995]: 875). Su questo punto cfr. Desideri ([1980]: 281): «fino a che l'Erlebnis (baudelairiano) diviene realmente Erfahrung».

${ }^{13}$ Cfr. Benjamin ([2007]: 874).

${ }^{14}$ Cfr. Benjamin ([2007]: 191).
} 
intrattiene col prezzo un rapporto allegorico: tra i due non c'è un nesso di derivazione calcolabile; il cartellino non rappresenta nulla di reale; una "scienza della merce" risulta impraticabile ${ }^{15}$. Il corso dei titoli di borsa si emancipa da ogni fondazione oggettiva: il loro valore attuale è l'effetto di un numero incalcolabile d'interazioni, incluse le scommesse sul loro andamento futuro ${ }^{16}$. Quale visione sintetica può tenere insieme questa esplosione di fatti? La pretesa di ricostruire sotto il profilo intellettuale la loro concatenazione sperimenta ben presto come «le cause si perdono sin dai primi anelli della catena nella vastità dell'infinito» (Musil [1986c]: 100).

Se la "fabbrica dell'intelletto" è stata dismessa, è ingenuo progettare di riammodernarla in modo che torni a produrre nuove compresse e pastiglie concettuali capaci di farci assimilare meglio un'epoca di per sé indigesta. Nessuno, nemmeno Husserl ${ }^{17}$, ritiene che a fronte di un lavoro pure estremamente rigoroso si possa guadagnare una quadratura del cerchio, una specie di restitutio in integrum. "Non si deve più costruire la realtà - dirà più tardi Adorno - perché si dovrebbe costruirla troppo a fondo» (Adorno [1970]: 22).

2. La strada è a senso unico: «siamo divenuti poveri» (Benjamin [2012]: 369) e lo svuotamento della vita interiore non troverà rimedio nel recupero o nel potenziamento dell'Io. La riflessione come "dinamica di consolidamento" della coscienza individuale poteva "funzionare", forse, nel XIX secolo ${ }^{18}$, ma nel passaggio attuale i suoi "schemi di comprensione" - il suo ideale di chiarezza e distinzione - vengono rimbalzati dal corso dell'accadere: «la vita gira intorno all'uomo e pensa, e danzando intesse per lui le relazioni che egli a fatica, e assai meno caleidoscopiche, affastella quando si serve della ragione» (Musil [1997]: 463). La collettività sognante dispone di un'auto-

\footnotetext{
${ }^{15}$ Cfr. Benjamin ([2007]: 743).

${ }^{16}$ Cfr. Simmel ([2019]: 281-282).

17 Sulla possibilità di vedere la "Crisi" già del tutto operante nelle Ricerche logiche cfr. M. Cacciari ([1975]: 213218).

${ }^{18}$ Cfr. Benjamin ([2007]: 433).
}

percezione estremamente più affinata, più sensibile, rispetto a quella dell'individuo sano e desto ${ }^{19}$, ancora filtrata dallo schermo della coscienza. «L'umanità si prepara a sopravvivere alla cultura» (Benjamin [2012]: 369) non nella forma della riflessione, ma come massa che incorpora e quasi inghiotte un'immane quantità di connessioni «al cui quotidiano aumentare lo sviluppo spirituale dei soggetti può tener dietro solo in modo incompleto, e con distacco sempre crescente» (Simmel [1995]: 53).

In un clima di frammentarietà inesprimibile il singolo osserva come «tutti gli altri che ne sanno di meno dicono sciocchezze a proposito del suo millimetro, eppure non può muoversi perché se si sposta anche di un solo micromillimetro dice sciocchezze anche lui»(Musil [1997]: 297). Col termine "intelligenza" s'intende l'abilità di presidiare il "proprio" millimetro quadrato di competenza. La prontezza nel denunciare l'incompetenza dei non adepti nulla sa degli altri pezzi del puz$z l e$, non ha strumenti per recepire le osservazioni o i comportamenti provenienti dal millimetro quadrato adiacente o limitrofo: li considera mere sciocchezze, stupidaggini. Dato il legame originario della parola "stupido" con un'idea generale di scarsa abilità, e considerando l'impenetrabilità dei diversi segmenti dell'intelligenza, non stupisce che «l'accusa reciproca di stupidità sia oggi così enormemente diffusa» (Musil [1986a]: 247).

Siccome "ogni intelligenza ha la sua stupidità» (Musil [1986a]: 246), chi si comporta in modo maldestro sarà chiamato stupido dagli specialisti del settore anche se è uno Hölderlin ${ }^{20}$. Al contempo, marchiando tutto ciò che non le va a genio col bollino della stupidità, l'intelligenza finisce in realtà per confessare il proprio corto circuito: l'incapacità d'intendere il discorso dell'altro si mani-

${ }^{19}$ Cfr. Benjamin ([2007]: 433): «il dormiente intraprende attraverso il suo corpo un viaggio macrocosmico: grazie allo straordinario affinamento della sua autopercezione, $\mathrm{i}$ rumori e le sensazioni dei suoi organi interni - pressione del sangue, movimenti intestinali, battito cardiaco e tensioni muscolari - che nellindividuo sano e sveglio si perdono nella risacca della buona salute».

${ }^{20} \mathrm{Su}$ questo punto cfr. Musil ([1986a]: 246). 
festa nel modo più lampante esattamente attraverso l'impiego dell'insulto. In quanto termine inflazionato, in grado di sostituire quasi ogni altra parola, «l'insulto non rappresenta ciò che dice, ma un misto di idee, di sentimenti e di intenti che l'insulto non può "esprimere", ma solo segnalare» (Musil [1986a]: 249), come se fosse un esito sintomatico. Secondo Freud il sintomo sarebbe «un sostituto di qualcos'altro che non ha avuto luogo» (Freud [1978]: 441).

A indicarci questa chiave di lettura è Musil, quando affianca l'insulto alle battute di spirito, alle frasi fatte, più in generale a tutte quelle formule che risultano sufficientemente imprecise e vaghe da riuscire a «sostituirsi, nell'uso, a interi gruppi di parole più calzanti, più oggettive, più esatte» (Musil [1986a]: 250). Rimpiazzare un'espressione più articolata con un surrogato a buon mercato è a sua volta stupido, ma non privo di valore: ci stiamo difendendo dalla possibile irruzione di uno stato di panico. Sopperiamo all'incapacità di formulare una proposta spirituale all'altezza - che dunque non ha avuto luogo sostituendola con un insulto e inflazionandolo, di modo che la quantità supplisca all'assenza di qualità. "Lo sfogo viene introdotto da queste parole: "Insomma, la cosa è troppo stupida". Ma la "cosa" troppo stupida è l'uomo che sta parlando» (Musil [1986]: 252). Come il fuoco da sbarramento che copre un obiettivo militare ${ }^{21}$ (o come la mosca che sbatte ripetutamente sulla finestra), investire gli altri con una "mitragliata" di insulti rivela una propria astuzia: moltiplicando i tentativi aumenta la possibilità, benché cento possano fare cilecca, che uno colpisca il bersaglio.

Sono anni senza sintesi ${ }^{22}$ : la situazione ambisce a un riepilogo spirituale che nessuno è in grado di fornire. Nelle pagine de L'uomo senza qualità recuperiamo una diagnosi: «l'ordine che si guadagna nel particolare si torna a perdere nell'insieme, cosicché abbiamo sempre più ordini e sempre meno ordine» (Musil [1997]: 429). Il panico da inadeguatezza viene coperto e rimpiazzato dall'in-

${ }^{21}$ Cfr. Musil ([1986a]: 251).

${ }^{22}$ Cfr. Musil ([1986d]: 186). sulto sintomatico. L'individuo è già stato assorbito dalla matrice-massa, pienamente integrato in essa: «secondo la massa il presupposto principale della felicità non è quello di risolvere le contraddizioni, bensì di farle sparire, come in un lungo viale spariscono i vuoti» (Musil [1997]: 737).

3. La stupidità segnala una "crisi della fiducia che fino ad oggi avevamo riposto nella natura umana» (Musil [1986a]: 253). Libertà e ragione ironizza Musil - non hanno più una bella cera: il binomio ereditato dell'età classica del cosmopolitismo tedesco si è sforzato invano di rimpiazzare il segreto millenario di un formidabile cemento sociale, ormai in fase di sgretolamento. "Finché si credeva nella religione si poteva buttar giù un buon cristiano o un devoto giudeo da qualunque piano della speranza o del benessere, egli sarebbe sempre caduto in piedi, per così dire sui piedi della propria anima» (Musil [1997]: 592): era previsto un "resto irrazionale" cui ricorrere qualora i conti del singolo non tornassero, quasi come se si potesse sempre richiedere un "credito di produzione". L'interazione tra le energie spirituali degli individui assicurata dallo sfondo religioso trova il proprio emblema - secondo Simmel ${ }^{23}$ - nelle parole dell'evangelista: «c'è posto per tutti nella casa del Padre» (Gv. 14, 2). Per tornare a Musil, «la Cacania era il primo paese al quale Iddio avesse tolto il credito, il piacere di vivere, la fiducia in se stesso» (Musil [1997]: 600).

Tradurre la dinamica della fede nella richiesta di un credito non delinea soltanto una vaga analogia tra il religioso e l'economico, ma attesta un vero e proprio passaggio di consegne, confermato dalle iscrizioni "non aes, sed fides" sulle monete di Malta o dal motto stampato sul dollaro "In God we trust". Data la sua indifferenza il denaro "costituisce il ponte più adatto e il migliore strumento d'intesa tra molte e diverse persone» (Simmel [2019]: 431). Per Arnheim è un simbolo, «il metodo oggi più sicuro per trattare tutti i rapporti» (Musil [1997]: 440): grazie al suo carattere sintetico-comprensivo, assicura la continu-

${ }^{23}$ Cfr. Simmel ([1993]: 70). 
ità dei processi. Se «la vera eticizzazione prodotta dal processo della civiltà consiste nel fatto che una quantità sempre maggiore di contenuti di vita viene oggettivata in forma transindividuale» (Simmel [2019]: 253), di fronte agli eventuali vuoti che si creano nei rapporti tra le cose e le relative valutazioni, «si inserisce il denaro al fine di evitare tale minacciosa interruzione» (Simmel [2019]: 106). La semplificazione dei rapporti si trasfigura in un'immane tensione oggettivante attorno a cui ruotano e si rinsaldano tutti i rapporti sociali.

Valéry immagina una malattia misteriosa in grado di attaccare e distruggere rapidamente $\mathrm{i}$ documenti, gli scritti, le banconote di tutto il globo: questa "tubercolosi della carta" - oggi forse un virus informatico - fulminerebbe di colpo l'intera vita sociale, riducendoci al campo dei sensi. «Supponete dunque che si indebolisca e sprofondi il supporto di questo supporto, e cioè la fede, la fiducia, il credito che concediamo a questa carta scritta e che le fornisce tutto il suo valore» (Valéry [1994a]: 76). L’interazione, la complicità tra denaro e stupidità viene in luce considerando non il supporto $^{24}$, bensì il supporto del supporto: dopo il tramonto della religione e la breve illusione della coppia libertà-ragione, la fiducia si presenta oggi per così dire dissociata in due componenti. Adoperiamo qui una formula analoga a quella mostrata da Blanchot quando distingueva il "si muore" dall' "io muoio" come due aspetti differenti della morte (cfr. Blanchot [1967]: 132-133). Anche la fiducia vivrebbe una scissione tra il suo "lato oggettivo", tutto assorbito nelle dinamiche del denaro, e il lato dei rapporti soggettivi, annebbiato nella stupidità.

L'azione fluidificante del denaro su un piano che Simmel chiamerebbe "cultura oggettiva" trova il proprio correlato a livello soggettivo quando «la stupidità avvolge questi bruti avvenimenti in una nebbia protettiva: la sua necessità, si sarebbe detto un tempo, è strutturale. Se i crepacci che si apro-

\footnotetext{
${ }^{24}$ In sé il tipo di supporto, oro, carta o bit risulta poco significativo, come si legge ne Le parole e le cose: «l'oro è prezioso perché moneta. Non il contrario» (Foucault [2007]: 193).
}

no tra $\mathrm{i}$ fatti non fossero riempiti da una bambagia di frasi fatte, se la schizoidia da laboratorio non fosse occultata dalla convinzione di fare il Bene, se il raziocinio devastatore non si ritenesse l'incarnazione del Buon Senso, se... - la macchina si paralizzerebbe» (Calasso [2007]: 763).

Quote sempre maggiori di fiducia si depositano nella forma-denaro: «il credito è diventato un'organizzazione impersonale e la fiducia ha perso quel carattere personale [...] il credito è diventato una forma tecnica di attività economica sostanzialmente priva di accentuazioni psicologiche» (Simmel [2019]: 411). Con lo sguardo rivolto all'europeizzazione del pianeta, Valéry auspicava un'Europa «perla della sfera, il cervello di un vasto corpo» (Valéry [1994b]: 35) - speranza delusa non perché oggi la perla si trovi oggi in un altro continente, ma in un senso più radicale: totalmente sradicata rispetto a ogni contesto locale, la perla è una "fiducia oggettiva" inafferrabile, gestaltlos, ma "intelligentissima", molto più di quanto possano cogliere le forme di razionalità alla portata di singoli individui - e con distacco sempre crescente. Svincolandosi dai limiti della fiducia in qualcuno, questa fiducia impersonale implementa processi autonomi di funzionamento, rispetto a cui la ridicola goccia dell' ${ }^{2}{ }^{25}$ è destinata a fare la figura della stupida.

Sul piano delle relazioni soggettive - il piano originario del suo consistere - la fiducia infiacchisce nella penombra della stupidità, in un'atmosfera di ottundimento che "non indica neppure un altrove del senso, ma lo elude - sovvertendo non il contenuto, ma l'intera pratica del senso» (Barthes [1985]: 56): l'altro è sempre uno stupido, tutti gli altri sono troppo stupidi. E stupido è chi sta parlando: quante volte ci diamo degli stupidi da soli; il "nostro" stesso io non si sottrae all'insulto.

"Denaro e intelligenza" - "singolarità e stupidità" agiscono in parallelo: "oggettività e impersonalità, da un lato, soggettività e inadeguatezza, dall'altro, sono imparentate tra loro» (Musil [1986a]: 259). Diventando sempre più intelligente, il denaro trasforma i singoli in stupidi. Il singolo

${ }^{25}$ Cfr. Musil ([1997]: 170). 
è percepito dai singoli come incapace, inaffidabile, indegno di quella fiducia che invece accordiamo al grande mezzo assoluto, "der irdisch got" come lo chiamava Hans Sachs. Il sentimento della fiducia trapassa via via sempre più in calcolo: «noi usciamo continuamente dallo stato di ciò che ha senso per entrare in quello del necessario e dell'appena sufficiente» (Musil [1997]: 1552). Per riassumere la tendenza che va imponendosi in misura crescente: ci si fida, ma io non mi fido.

4. Alla concentrazione d'intelligenza nel denaro corrisponde una sempre maggiore stupidità del singolo: il salto tecnologico degli ultimi decenni non ha fatto che aumentare tale polarizzazione. Il livello di articolazione, di complessità e di specializzazione che la vita ormai comporta sul piano sociale, economico e politico tende a trasformare la stupidità occasionale di cui ciascuno, prima o poi, è protagonista in una specie di stupidità funzionale, e dunque costitutiva della collettività stessa. "Possiamo dire senz'altro che la stupidità e l'intelligenza dipendono tanto dall'intelletto quanto dal sentimento» (Musil [1986a]: 254): già Simmel aveva rilevato una separazione tra intelletto e strati profondi della personalità ${ }^{26}$. Dunque si può essere stupidi in due modi: o perché troppo intelligenti, o perché deboli d'intelletto.

$\mathrm{Da}$ un lato troviamo una sorta di "stupidità intelligente", ovvero di un'intelligenza che risulta troppo stupida perché si sforza di essere "solo intelligenza", intelligenza allo stato puro: non è vera mancanza d'intelligenza, ma «un fallimento dell'intelligenza, che si è arrogata dei compiti che non erano i suoi» (Musil [1986a]: 257). Nessuno di noi, in effetti, si comporta sempre con tutta l'intelligenza necessaria: «un comportamento completamente oggettivo è impossibile sia viverlo, sia pensarlo» (Musil [1986a]: 259). L'effetto complessivo

${ }^{26}$ Cfr. Simmel ([1995]: 36-37): «l'intelletto è la più adattabile delle nostre forze interiori: per venire a patti con i cambiamenti e i contrasti dei fenomeni non richiede quegli sconvolgimenti e quei drammi interiori che la sentimentalità a causa della sua natura conservatrice, richiederebbe necessariamente per adattarsi a un ritmo analogo di esperienze». dello sforzo di adeguamento da parte del singolo ai parametri di ciò che potremmo chiamare l'intelligente necessità oggettiva "contribuisce a vivacizzare la vita spirituale, ma soprattutto la rende incostante e sterile» (Musil [1986a]: 257). In altre parole, questa stupidità è la più letale delle malattie dello spirito, una vera patologia della cultura.

Sull'altro fronte, vi è una stupidità per così dire ingenua, un po' dura di comprendonio, che non è nemmeno in grado di rispondere alle sollecitazioni in modo "intelligente": una stupidità onesta e schietta, una genuina incapacità di reagire automaticamente agli stimoli. Attribuendo importanza a elementi "oggettivamente" superflui, a circostanze accessorie, finisce in realtà per rivelare, nella sua autentica idiozia, qualcosa di poetico: «la stupidità schietta è una vera artista» (Musil [1986a]: 256).

\section{BIBLIOGRAFIA}

Adorno, T.-W., 1970: Dialettica negativa, tr. it. di C.A. Donolo, Einaudi, Torino.

Barthes, R., 1985: Lovvio e l'ottuso. Saggi critici III, tr. it. di C. Benincasa, G. Bottiroli, G.P. Caprettini, D. De Agostini, L. Lonzi, G. Mariotti, Einaudi, Torino.

Benjamin, W., 2007: I "passages" di Parigi, a cura di R. Tiedeman e E. Ganni, Einaudi, Torino.

Benjamin, W., 2012: Esperienza e povertà, in Aura e choc. Saggi sulla teoria dei media, a cura di A. Pinotti e A. Somaini, Einaudi, Torino.

Blanchot, M., 1967: Lo spazio letterario, Einaudi, Torino.

Cacciari, M., 1975: Di alcuni motivi in Walter Benjamin, "Nuova Corrente" 67, pp. 209-243.

Calasso, R., 2007: La guerra perpetua, postfazione a Kraus, K., Gli ultimi giorni dell'umanità, Adelphi, Milano, pp. 755-779.

Desideri, F., 1980: Walter Benjamin. Il tempo e le forme, Editori Riuniti, Roma.

Foucault, M., 2007: Le parole e le cose. Un'archeologia delle scienze umane, tr. it. di E. Panaitescu, Rizzoli, Milano.

Freud, S., 1978: Opere 1915-1917. Introduzione alla psicoanalisi e altri scritti, a cura di L.C. Musatti, Boringhieri, Torino. 
Kraus, K., 2007: Gli ultimi giorni dell'umanità, a cura di E. Braun e M. Carpitella, Adelphi, Milano.

Musil, R., 1986a: La stupidità, in Sulla stupidità e altri scritti, tr. it. di A. Casalegno, Mondadori, Milano.

Musil, R., 1986b: L'Europa abbandonata a se stessa, in Musil, R., Sulla stupidità e altri scritti, Mondadori, Milano.

Musil, R., 1986c: Spirito ed esperienza. Note per $i$ lettori scampati al tramonto delloccidente, in Sulla stupidità e altri scritti, Mondadori, Milano.

Musil, R., 1986d: Libri e letteratura, in Musil, R., Sulla stupidità e altri scritti, Mondadori, Milano.

Musil, R., 1997: L'uomo senza qualità, a cura di A. Frisé, Einaudi, Torino.

Musil, R., 2014: L'uomo tedesco come sintomo, a cura di F. Valagussa, Pendragon, Bologna.

Simmel, G., 1993: Per una sociologia della religione, in Saggi di sociologia della religione, tr. it. di M. Marroni, Borla, Roma.

Simmel, G., 1995: Le metropoli e la vita dello spirito, a cura di P. Jedlowski, Armando, Roma.

Simmel, G., 1997: Intuizione della vita. Quattro capitoli metafisici, a cura di G. Antinolfi, ESI, Roma.

Simmel, G., 2019: Filosofia del denaro, a cura di A. Cavalli e L. Perucchi, Ledizioni, Milano.

Valéry, P., 1994a, La politica del pensiero, in Valéry, P., La crisi del pensiero e altri "saggi quasi politici”, tr. it. N. Agosti, il Mulino, Bologna.

Valéry, P., 1994b: La crisi del pensiero, in Valéry, P., La crisi del pensiero e altri "saggi quasi politici", il Mulino, Bologna.

Valéry, P., 2008: L'idea fissa, a cura di V. Magrelli, Adelphi, Milano. 